\title{
Giant Dielectric Permittivity Observed in Pb-Based Perovskite Ferroelectrics
}

\author{
Bog-Gi Kim,* Seong M. Cho, Tea-Yong Kim, and Hyun M. Jang \\ Department of Material Science and Engineering, Pohang University of Science and Technology (POSTECH), San 31, \\ Hyoja-dong, Nam-gu, Pohang 790-784, Korea
}

(Received 27 September 2000)

\begin{abstract}
Giant dielectric permittivity was observed in La-modified $\mathrm{PbTiO}_{3}$ (PLT) with $A$-site vacancy. The observed values of PLT with $A$-site vacancy are 1 order of magnitude larger than those of relaxor ferroelectrics. The giant relative dielectric permittivity, coupled with a low dielectric loss $(\tan \delta \approx 0.03)$ of the PLT, potentially makes it one of the most promising materials for numerous modern technological applications.
\end{abstract}

A technological breakthrough is made, more often than not, by the discovery of a new material with innovative properties. In the case of ferroelectrics, the discovery of lead zirconia-titanate, $\mathrm{Pb}(\mathrm{Zr}, \mathrm{Ti}) \mathrm{O}_{3}[1,2]$, opened a door to the technological renovations and applications of numerous modern transducers that include the future ferroelectric random access memory devices [3] and sensors/actuators used in micro-electro-mechanical systems (MEMS) [4,5]. Here, we report the development of $\mathrm{Pb}$-based perovskite ferroelectrics with supreme dielectric properties. The relative dielectric permittivity of $15 \%$ La-modified $\mathrm{PbTiO}_{3}$ (PLT) with the $A$-site vacancy is approximately 450000 near the transition temperature and is greater than 65000 even at room temperature. The present development is truly remarkable in a sense that these values are greater than those of any other high-permittivity dielectric material by 1 order of magnitude. The giant relative dielectric permittivity, coupled with a low dielectric $\operatorname{loss}(\tan \delta \approx 0.03)$ of the PLT potentially makes it one of the most promising materials for numerous modern technological applications, including energy storage applications, thin film microactuators in MEMS devices, and the future Gb-DRAM (dynamic random access memory) dielectric layers.

Lead titanate, $\mathrm{PbTiO}_{3}$, has long been regarded as a typical ferroelectric perovskite. Because of its structural, chemical simplicity, in addition to its displacive nature exhibited during the phase transition, extensive studies have been conducted on the characteristics of $\mathrm{PbTiO}_{3}$, especially on the nature of the "soft" phonon mode associated with the displacive first-order ferroelectric transition [6,7]. The addition of lanthanum (La) enhances diffuse characteristics in the temperaturedependent dielectric response near the phase transition point and decreases the Curie temperature significantly. The PLT has been regarded as an ideal model system for the understanding of the nature of relaxor ferroelectricity and the spontaneous relaxor-normal ferroelectric transition recently discovered in several $\mathrm{Pb}$-based perovskites [8-11]. This is because a change in the nature of ferroelectricity from normal to relaxor behavior can be induced by the addition of La above a certain critical concentration [8].

It is generally believed that trivalent La ions substitute for $\mathrm{Pb}$ ions, requiring a charge compensation process in the PT-based perovskite lattice. According to the early study done by Hennings [12], this charge compensation can be done either by the formation of the $A$-site vacancy ( $\mathrm{Pb}$ site) or by the formation of the $B$-site vacancy (Ti site). $A$-site vacancy can give a strain field to Ti-O octahedral, whereas $B$-site vacancy breaks Ti-O octahedral. The $B$-site vacancy is primarily responsible for the manifestation of relaxor behavior [13]. The control of these defect processes should be considered as an important step and improvement on the electrical properties of $\mathrm{Pb}$-based perovskite ferroelectrics. However, because of the problem encountered in the processing, it is extremely difficult, even though not impossible, to arbitrarily adjust the relative concentration of these two distinct types of defects.

Another problem associated with the processing of $\mathrm{Pb}$-based perovskites is the $\mathrm{PbO}$ evaporation that occurs during the sintering stage. Since the evaporation of $\mathrm{PbO}$ produces the $A$-site vacancies, the control over these two distinct defect processes and the suppression of the $\mathrm{PbO}$ evaporation are not independent but are closely interrelated with each other. Until now, none of the investigators have successfully removed these problems that inevitably occur in the course of the fabrication of $\mathrm{Pb}$-based perovskites. Therefore, most electrical and dielectric properties of $\mathrm{Pb}$-based perovskite dielectrics reported in the literature are not likely to be their inherent properties and, thus, are subject to further careful investigations. In view of these, it is highly desirable to establish a new processing scheme in which the above-mentioned two problems are effectively removed.

The PLT employed in the present study has been carefully designed such that it basically contains the $A$-site vacancy only. The composition of PLT having the $A$-site vacancy only (PLT- $A$ ) can be represented by $\left(\mathrm{Pb}_{1-3 x / 2} \mathrm{La}_{x}\right) \mathrm{TiO}_{3}$, whereas it can be denoted by $\mathrm{Pb}_{1-x} \mathrm{La}_{x} \mathrm{Ti}_{1-x / 4} \mathrm{O}_{3}$ if a specimen exclusively has the 
$B$-site vacancy (PLT- $B$ ). For the processing of the PLT- $A$, the mixed powder with an exact stoichiometric ratio, $x=0.10,0.15,0.20,0.23,0.25$ (abbreviated as PLT-A100x), was calcined at 850 for $4 \mathrm{~h}$. The cold isostatically pressed specimens were sintered at 1250 for $2 \mathrm{~h}$ in an air atmosphere. Contrary to conventional processing of $\mathrm{Pb}$-based perovskites, the $\mathrm{PbO}$-rich atmosphere was not maintained during the sintering. This effectively eliminates the problem caused by the $\mathrm{PbO}$ absorption into the specimen and, thus, minimizes the formation of the $B$-site vacancy. By analyzing micro-Raman signal as a function of depth, we can confirm that $\mathrm{PbO}$ evaporation occurs within $3 \mathrm{~mm}$ from the surface. To obtain the PLT- $A$ system with a minimum $\mathrm{PbO}$ evaporation, a surface region $(3 \mathrm{~mm})$ was removed after the sintering. Synchrotron $\mathrm{x}$-ray diffraction confirmed a single perovskite phase with the absence of minority phases. Extended x-ray absorption fine structure studies and $\mathrm{x}$-ray photoemission spectroscopy measurement determined the valence of Ti to be $4+$. The inductively coupled plasma analysis confirmed that all PLT samples had only an $A$-site vacancy to within detection limits. A scanning electron microscope showed that each grain was about $2-6 \mu \mathrm{m}$ in size without open pores.

A dielectric constant measurement was conducted with a block of $6 \mathrm{~mm} \times 6 \mathrm{~mm} \times 2.5 \mathrm{~mm}$ in size. The polished surfaces were ultrasonically cleaned with methanol to remove dust particles then electroded by gold sputtering. Silver paint was used on top of the gold electrode. The dielectric response was measured using a Hewlett-Packard 4194A gain phase analyzer, with a frequency range from $150 \mathrm{~Hz}$ to $15 \mathrm{MHz}$ and oscillation voltage of $0.5 \mathrm{~V}$. All of the measurements were performed on a cooling run using a rate of $2{ }^{\circ} \mathrm{C} / 5 \mathrm{~min}$.

The plots of relative dielectric permittivity versus temperature for the PLT- $A$ specimens containing various amounts of $\mathrm{La}$ are presented in Fig. 1 for a frequency of $1.5 \mathrm{kHz}$. As shown in the figure, the relative dielectric permittivity of the $15 \%$ La-substituted specimen is 450000 near the phase transition temperature and is higher than 65000 even at room temperature. The highest relative permittivity value observed in $\mathrm{PbMg}_{1 / 3} \mathrm{Nb}_{2 / 3} \mathrm{O}_{3}$ (PMN) and $\mathrm{PbZn}_{1 / 3} \mathrm{Nb}_{2 / 3} \mathrm{O}_{3}$ single crystal is between 20000 and 30000 at $T_{\max }$, respectively $[14,15]$. The relative permittivity values of PMN-type polycrystalline ceramics are lower than those of single crystals, which are about 15000 at $T_{\max }$. Thus, the present discovery is truly remarkable in a sense that these permittivity values are greater than those of any other dielectric material by 1 order of magnitude (i.e., 450000 vs 15000 ).

As can be noticed from Fig. 1, $T_{\max }$ gradually decreased with increasing content of La. As a result of this, the room-temperature permittivity increases with the $\mathrm{La}$ content, from 30000 for PLT-A10 to 160000 for PLT-A25. Examination of Fig. 1 also indicates that the deviation from the Curie-Weiss behavior increases with increasing

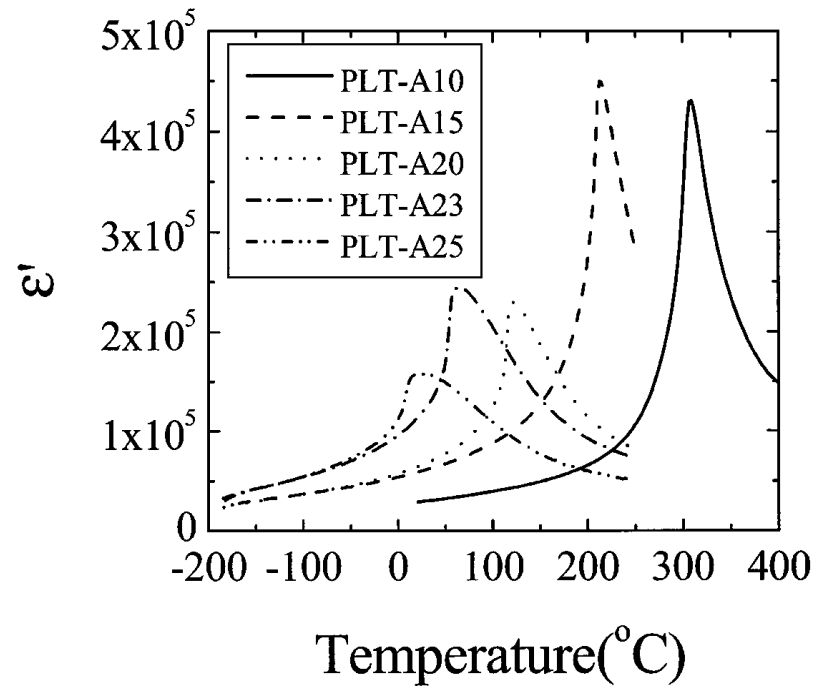

FIG. 1. Temperature dependence of real part of dielectric constant in various La concentration of PLT- $A$ (lanthanum modified lead titanate with $A$-site vacancy). Note that numbers represent lanthanum concentrations.

content of La, further suggesting that the addition of lanthanum not only decreases the phase transition temperature but also enhances the diffuse phase transition characteristics.

The frequency-dependent dielectric permittivity of the PLT-A20 is plotted in Fig. 2 as a function of temperature. The real part of the dielectric permittivity increases from 65000 at room temperature to 250000 near the phase transition point. The rapid increase in the dielectric loss near $120^{\circ} \mathrm{C}$ can be attributed to the para-ferro phase transition. This phenomenon is called "the critical slowing down" and is common to a ferroelectric system near the phase transition point [16]. As shown in Fig. 2, the frequency-dependent dielectric dispersion is essentially absent between the phase transition point and the liquid nitrogen temperature. This suggests that the observed huge dielectric permittivity of the PLT- $A$ is not caused by either the space-charge or the surface barrier layer.

The imaginary part of the complex dielectric permittivity indicates an occurrence of the dipolar freezing below $-100{ }^{\circ} \mathrm{C}$. A strong dielectric dispersion at this low temperature region is similar to the freezing of the dipole glass $[17,18]$. This kind of freezing behavior was also observed in glass-forming liquid [19] and relaxor ferroelectrics [20-23]. As indicated in Fig. 2, the onset of a rapid increase in the dielectric loss systematically moves to a higher temperature region with increasing frequency. This suggests that, below a certain critical temperature, the dipoles are frozen or ordered in their potential-energy wells. More detailed results and the analysis on the nature of dipolar freezing of the PLT- $A$ will be given in a forthcoming paper. 


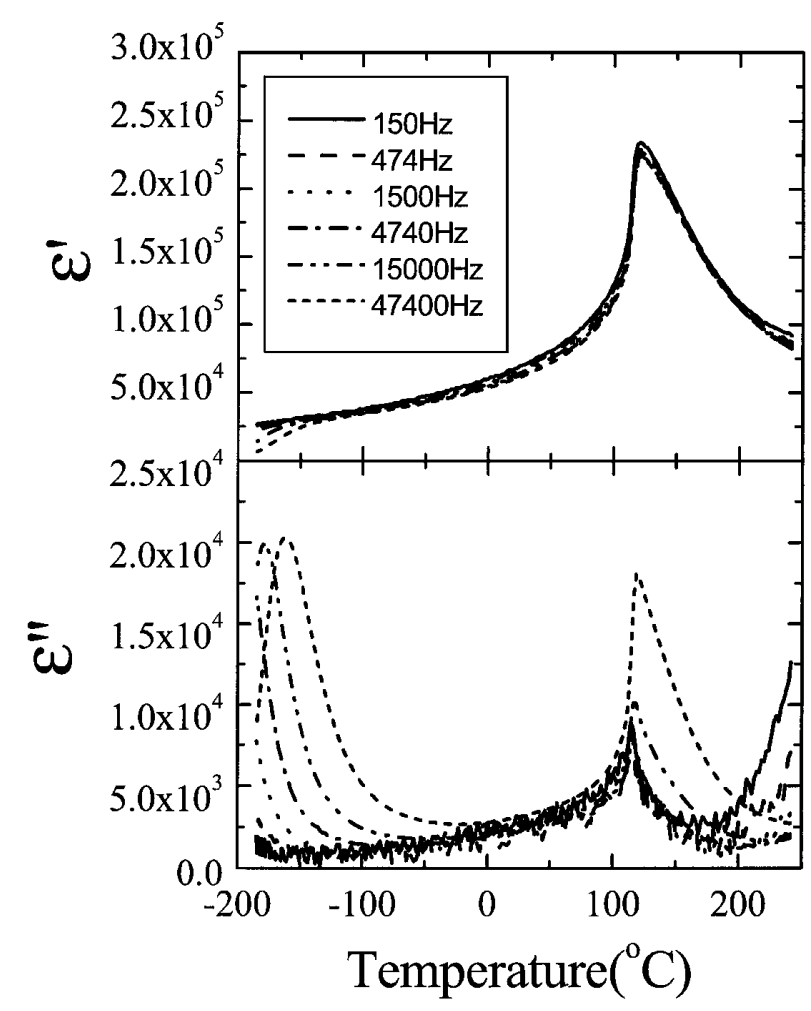

FIG. 2. (top) Frequency dependence of real part of dielectric constant in PLT-A20 (20\% lanthanum modified lead titanate with $A$-site vacancy). (bottom) Frequency dependence of imaginary part of dielectric constant in PLT-A20. Anomaly around $120{ }^{\circ} \mathrm{C}$ is due to critical slowing down. Dielectric dispersion around $-150{ }^{\circ} \mathrm{C}$ is due to dipolar relaxation.

We have reported the discovery of innovative dielectric properties in the La-modified $\mathrm{PbTiO}_{3}$ with the $A$-site vacancy. This development is possible by effectively eliminating the formation of the $B$-site vacancy while minimizing the $\mathrm{PbO}$ variation in the specimen. The giant relative dielectric permittivity and the low loss factor ( $\tan 0.03$; Fig. 2), coupled with the thermostability in the dielectric response of the PLT- $A$, potentially make it one of the most promising materials for the technological application: (a) high density energy storage, (b) thin-film devices such as MEMS and Gb-DRAM, and (c) multilayer ceramic capacitors having a huge dielectric capacitance.
This work was supported by KISTEP through the NRL program and by the POSTECH through "POSTECH/BSRI Special Fund-1999."

* Now at Department of Physics and Astronomy, Rutgers University, Piscataway, NJ 08854.

[1] B. Jaffe, R. S. Roth, and S. Maezullo, J. Appl. Phys. 25, 809 (1954).

[2] B. Jaffe, W. R. Cook, and H. Jaffe, Piezoelectric Ceramics (Academic, New York, 1971), Chap. 7.

[3] J. F. Scott and C. A. Araujo, Science 246, 1400 (1989).

[4] J. D. Olivas and S. Bolin, JOM 50, 38 (1998).

[5] S. Ballandras et al., Sens. Actuators A 58, 265 (1997).

[6] G. Shirane, J. D. Axe, J. Harada, and J. P. Remeika, Phys. Rev. B 2, 155 (1970).

[7] G. Burns and B. A. Scott, Phys. Rev. B 7, 3088 (1973).

[8] X. Dai, Z. Xu, and D. Viehland, J. Appl. Phys. 79, 1026 (1996).

[9] X. Dai, A. DiGiovanni, and D. Viehland, J. Appl. Phys. 74, 3399 (1993).

[10] F. Chu, I. M. Reaney, and N. Setter, J. Appl. Phys. 77, 1671 (1995).

[11] M. S. Yoon and H. M. Jang, J. Appl. Phys. 77, 3991 (1995).

[12] D. Hennings, Mater. Res. Bull. 6, 329 (1971); D. Hennings and G. Rosenstein, Mater. Res. Bull. 7, 1505 (1972).

[13] T.-Y. Kim and H. M. Jang, Appl. Phys. Lett. 77, 3824 (2000).

[14] L. E. Cross, Ferroelectrics 76, 241 (1987).

[15] J. Kuwata, K. Uchino, and S. Nomura, Ferroelectrics 22, 863 (1979).

[16] T. Mitsui, O. Tatsuzaki, and E. Nakamura, Introduction to the Physics of Ferroelectrics, Ferroelectricity and Related Phenomena Vol. 1, edited by I. Lefkowitz and G. W. Taylor (Gordon and Breach, New York, 1976), Chap. V.

[17] E. Courtens, Phys. Rev. Lett. 52, 69 (1984).

[18] E. Courtens, Ferroelectrics 72, 229 (1987).

[19] P. K. Dixon et al., Phys. Rev. Lett. 65, 1108 (1990).

[20] D. Viehland, S. J. Jang, L. E. Cross, and M. Wuttig, J. Appl. Phys. 68, 2916 (1990).

[21] D. Viehland, J. F. Li, S. J. Jang, and L. E. Cross, Phys. Rev. B 43, 8316 (1991).

[22] V. Westphal, W. Kleemann, and M. D. Glinchuk, Phys. Rev. Lett. 68, 847 (1992).

[23] R. Blinc et al., Phys. Rev. Lett. 83, 424 (1999); R. Pirc and R. Blinc, Phys. Rev. B 60, 13470 (1999). 